\title{
Adaptive Position based Reliable Routing Protocol (APBRRP) for Mobile Ad Hoc Networks
}

\author{
B. Narasimhan \\ Assistant Professor, \\ Department of Computer Technology, \\ Dr. N.G.P. Arts and Science College, \\ Dr.N.G.P. Nagar, Kalapatti Road, \\ Coimbatore - 641048.
}

\author{
R. Vadivel \\ Assistant Professor, \\ Department of Information Technology, \\ School of Computer Science and Engg, \\ Bharathiar University, \\ Coimbatore - 641046.
}

\begin{abstract}
Routing packets in mobile ad hoc networks has been considered to a great extent, even though the hypothesis on full connectivity is generally not valid in a real time system which means that a practical routing protocol ought to handle intermittent connectivity and the absence of end-to-end connections. In this paper, we propose a location aware routing mechanism called adaptive position based routing protocol for mobile ad hoc networks, which is enhanced with a positioning service which well suits real time mobile ad hoc networks. In order to achieve low overhead, APBRRP uses a beaconless strategy combined with a position-based resolution during forwarding packets. Also the proposed protocol is combined with a local database holding mechanism which is updated using route overhearing. The proposed routing protocol APBRRP is compared with the reactive AODV routing protocol by performance metrics delay, overhead and delivery ratio. By the extensive simulation results using NS2 the results proved that our proposed APBRRP outperforms AODV.
\end{abstract}

\section{INTRODUCTION}

Mobile ad hoc networks have attached a lot of attention due to the popularity of mobile devices and the advances in wireless communication technologies [10]. A mobile ad hoc network is a peer-to-peer multihop mobile wireless network that has neither a fixed infrastructure nor a central server.

The nodes present in mobile ad hoc network are moving around and has the characteristic of acting as router, and also communicates with each other. Location based routing [2] uses location information to forward data packets, in a hopby-hop routing fashion. Greedy forwarding is used to select next hop forwarder with the largest positive progress toward the destination while void handling mechanism is triggered to route around communication voids [3].

No end-to-end routesneed to be maintained, leading to GR's high efficiency and scalability. However, GR is very sensitive to the inaccuracy of location information [4]. In the operation of greedy forwarding, the neighbor which is relatively far away from the sender is chosen as the next hop. If the node moves out of the sender's coverage area, the transmission will fail. In GPSR [5] (a very famous geographic routing protocol), the MAC-layer failure feedback is used to offer the packet another chance to reroute.

However, our simulation reveals that it is still incapable of keeping up with the performance when node mobility increases. In fact, due to the broadcast nature of the wireless medium, a single packet transmission will lead to multiple receptions. If such transmission is used as backup, the robustness of the routing protocol can be significantly enhanced. In order to acquire the internodes loss rates, periodic network-wide measurement is required, which is impractical for mobile environment. As mentioned in [9], the batching used in these protocols also tends to delay packets and is not preferred for many delay sensitive applications.

\section{RELATED WORKS}

In order to make geographical routing protocol to be triumphant, it is supposed to be supplemented by a position service which is capable enough to provide position information for all possible destination nodes. There exists a considerable corpse of research which treats position services for mobile ad hoc networks (The survey paper of Das et al. [1])

The theoretically trouble-free protocols preserve no knowledge about how the nodes move, where they are, or the nodes they have previously encountered. Two such theoretically simple protocols are: 1) randomized routing [4], where a packet randomly jumps around between nodes until it reaches the destination, and 2) epidemic routing [5], where every node in the network receives a copy of a packet. Another conceptually simple scheme, but one that actively uses node mobility and limits its overhead, is spray and wait [2]. In spray and wait, a packet is distributed to a limited number of nodes that hold on to the packet until they (potentially) meet the destination.

Cerf et al. have portrayed architecture for DTNs [3], in which a large and heterogeneous system transports data bundles between custodians that temporarily store the bundles until they can be forwarded again. The key difference between the authors [3] view view of a DTN and the proposed view of an IC-MANET is in the size and diversity of the systems. It can be notified that an IC-MANET as a relatively homogeneous system with a relatively modest spatial distribution. This difference in system properties leads to the proposal that the routing should be done on the network.

In common most of the proposed MANET routing protocols transfer packets between nodes using a link-layer unicast transfer mode. This condition enables error correction at the link layer, but it does not exploit the broadcast nature of wireless transmissions. In opportunistic routing (OR) [6], a packet is sent in a broadcast mode to several eligible forwarders, and the best forwarder that received the packet will continue to forward it.

The challenge in OR is how knowledge about the best forwarder can be distributed. One way of doing the selection of the forwarder is by geographical selection, which is an 
approach taken in contention-based forwarding (CBF) [7] and beaconless routing (BLR) [8]. Our proposed protocol builds on these principles and extends them to meet the requirements of an IC-MANET.

Because of the disconnected nature of the network, there will be similar problems with delays as for the mapping-based location services. One protocol that attempts to limit the cost of a location request by having a proactive component is Brownian gossip [9]. In Brownian gossip, nodes exchange information on previous encounters when two nodes meet. This information is used to guide a location request toward the destination node's position.

\section{ADAPTIVE POSITION BASED RELIABLE ROUTING PROTOCOL (APBRRP) FOR MANET}

APBRRP is a geographical routing protocol for sporadically attached mobile ad hoc networks which coalesces geographical routing and the store-carry-forward principle. APBRRP is a beaconless protocol. It uses greedy packet forwarding when possible. When greedy forwarding is not possible i.e the nodes could not communicate with each other, the node that holds the packet (the curator) waits until node mobility makes it possible to resume greedy forwarding.

To forward a message to the destination node, a curator node simply broadcasts the message. All mobile nodes within a preferably forwarding area are eligible to forward the packet and are called tentative curator nodes. All tentative curator nodes sets a delay timer specific for each mobile node, and the node whose delay timer expires first is the selected new curator node. After becoming a curator node, the node forwards the message in the same manner as the previous curator node. The old curator node which sent the message and most other tentative curator nodes will overhear this transmission and conclude that a new node has taken over custody of the packet. When no such transmission is known, the current curator node repeats again and again the broadcast of the message with a node delay interval awaiting a new curator node becomes available due to node mobility.

The rebroadcast time is assigned randomly for each transmission between values of stipulated range. The values are selected since forwarding opportunities are not to be missed. Also in order to limit the wastage of bandwidth the values are selected in random fashion. It is possible that not all nodes in the forwarding area will overhear the broadcast made by the new custodian, thereby producing packet duplicates.

This case will not only increase the load in the system but will enable the exploration of multiple paths to the destination as well. When the paths of two copies cross, only one copy will continue to be forwarded. To prevent a packet from indefinitely trying to find a path to its destination, all packets have a time to live TTL expressed as duration. When the TTL expires, a packet is deleted by its custodian. The forwarding area can have different characteristics, but it should be designed in such a way that progress toward the destination is guaranteed. One of the most significant properties of APBRRP is that for all nodes within the forwarding area to hear each other's transmissions.
This phenomenon will reduce the risk of tentative curator nodes failing to receive the packet transmitted by the new curator node.

The longest distance between two points within these shapes must be the preceptor radio range of the node(s). If overhearing is not a critical property and we want to maximize the probability of finding a new curator node, then the forwarding area should include all nodes that guarantee progress toward the destination node.

To avoid very small hops and to supply for inaccuracies in the location service, e.g., the Global Positioning System (GPS), a minimum forward distance may be discreet. All these forwarding areas can be used in APBRRP as a parameterized input. In this research work, we have chosen the packet progress forwarding area, and we will return to the basis. The delay timer for each mobile node can be set based on many principles, where two natural ones are to favour short hops or long hops toward the destination node.

Short hops are beneficial when much data will be exchanged between the nodes, because the transfer probability is higher with a shorter distance. The snag of APBRRP is that more hops may be created resulting in higher overhead. Long hops will reduce the number of hops, by which resulting the snag of the transfer reliability between distant nodes is lesser.

Another significant property is the proposed delay timer function which does not take the direction of node movement into account, although this condition would have been feasible. The main reason is that, even if the next curator node might move in the wrong direction, anticipate is that it can forward the packet to a node closer to the destination node. Another reason is that node directions are unstable, and a node might turn and move toward the destination node. For these reasons, a packet is always forwarded toward the destination node, even if it, in some cases, might be returned to the old curator node due to node movement.

In order to stop additional transmission of a packet by curator nodes and tentative curator nodes when ithas been delivered to the destination, an acknowledgement packet (ack) is sent by the destination node during reception of packets. All nodes that hear an ack will store the acknowledgement information until the packet times out based on TTL.

If a node receives a packet for which it previously has received an acknowledgement, then it broadcasts an acknowledgement to stop the transmission of the packet. Acknowledgements are not intended to reach the source; they are only intended to prevent further forwarding attempts by nodes holding the acknowledged packet.

To administer the inaccuracies intrinsic in MANET position service, APBRRP inquires the location service at each packet hop, and if more accurate (more recent) location data are available, then the routed packet gets updated by the algorithm.

This way, the quality of the location data is incrementally improved as the packet approaches the destination node. Also to improve the quality of the position data in the location service, our proposed APBRRP provides it with the position data available contained in received packets. 


\section{SIMULATION SETTINGS, PERFORMANCE METRICS AND SIMULATION RESULTS}

\subsection{SIMULATION SETTINGS}

Table 1. Simulation Settings

\begin{tabular}{|l|l|}
\hline No. of Nodes & 100 \\
\hline Area Size & 1500 X $1500 \mathrm{Km}^{2}$ \\
\hline MAC & $802.11 \mathrm{~b}$ \\
\hline Radio Range & 250 meters \\
\hline $\begin{array}{l}\text { Simulation } \\
\text { Time }\end{array}$ & 100 seconds \\
\hline $\begin{array}{l}\text { Traffic } \\
\text { Source }\end{array}$ & CBR \\
\hline Packet Size & 512 KB \\
\hline $\begin{array}{l}\text { Mobility } \\
\text { Model }\end{array}$ & Random \\
\hline Waypoint Model \\
\hline
\end{tabular}

\subsection{PERFORMANCE METRICS}

Control overhead: The control overhead is defined as the total number of routing control packets normalized by the total number of received data packets.

Average end-to-end delay: The end-to-end-delay is averaged over all surviving data packets from the sources to the destinations.

Average Packet Delivery Ratio: It is the ratio of thenumber of packets received successfully and the total numberof packets sent.

\subsection{RESULTS}

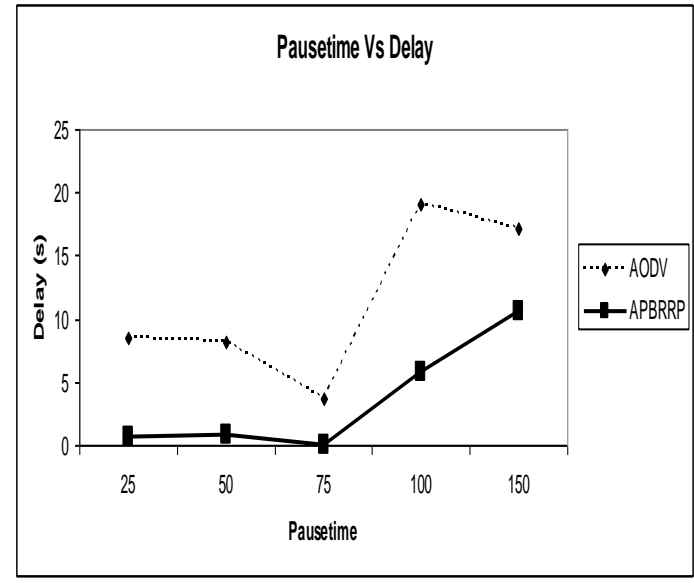

Figure 3. Pausetime Vs Delay
In Figure 1 it is observed that our proposed APBRRP consumes lesser overhead when compared to AODV routing protocol. From Figure 2 it is observed that our proposed APBRRP consumes better packet delivery ratio when compared to AODV routing protocol. In Figure 3 it is clearly visible that our proposed APBRRP consumes lesser delay when compared to AODV routing protocol.

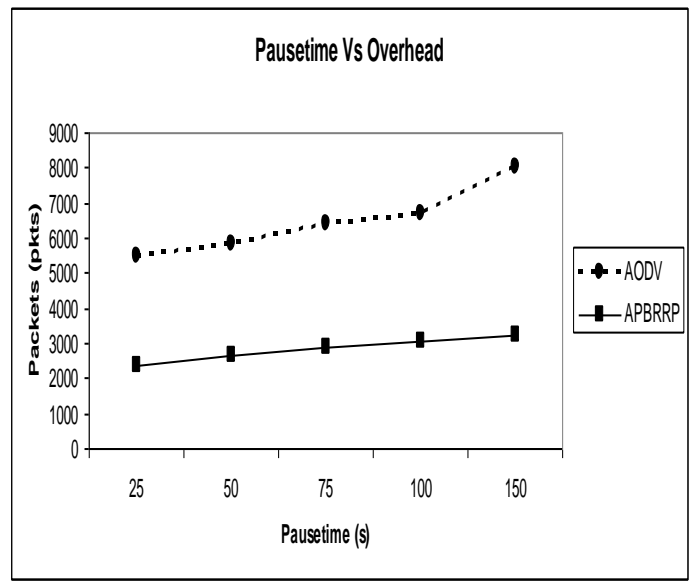

Figure 1. Pausetime Vs Overhead

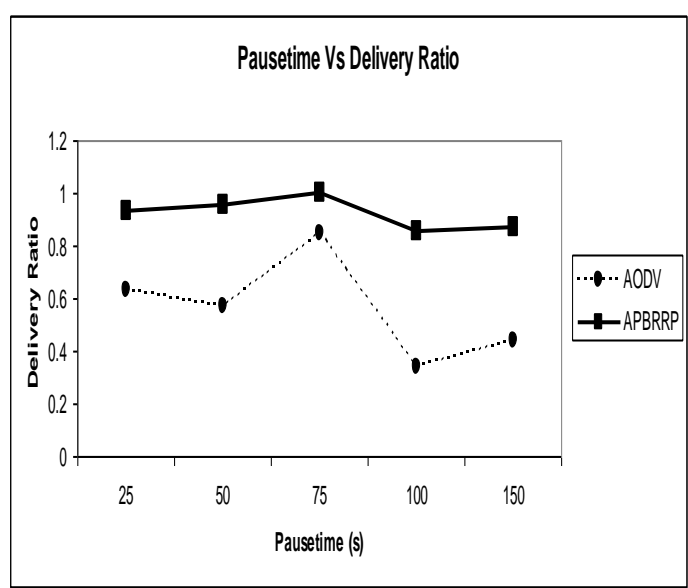

Figure 2. Pausetime Vs Delivery Ratio

\section{CONCLUSION}

In this paper, we proposed a location aware routing mechanism namely adaptive position based routing protocol (APBRRP) for mobile ad hoc networks. APBRRP is enhanced with a positioning service which well suits real time mobile ad hoc networks. In order to achieve low overhead, APBRRP uses a beaconless strategy combined with a position-based resolution during forwarding packets.

Also the proposed protocol is combined with a local database holding mechanism which is updated using routing overhearing. The proposed routing protocol APBRRP is compared with conventional AODV routing protocol using NS2 by choosing performance metrics delay, overhead and delivery ratio.

The NS2 simulation results proved that our proposed APBRRP outperforms AODV by increased packet delivery ratio with reduced delay and overhead packets. 


\section{ACKNOWLEDGEMENTS}

The first author thanks the management of Dr.N.G.P. Educational Institutions for providing career opportunity as an Assistant Professor in the Department of Computer Technology at Dr. N.G,P. Arts and Science College, Coimbatore. The second author thanks UGC for allocating Minor Research Project grant during Feb 2011 to Feb 2013 Grant Number 39-947/2010 (SR).

\section{REFERENCES}

[1] S. M. Das, H. Pucha, and Y. C. Hu, "Performance comparison of scalable location services for geographic ad hoc routing," in Proc. IEEE 24th Annu. Joint Conf. IEEE Comput. Commun. Soc., 2005, pp. 1228-1239.

[2] T. Spyropoulos, K. Psounis, and C. S. Raghavendra, "Spray and wait: An efficient routing scheme for intermittently connected mobile networks," in Proc. ACM SIGCOMM Workshop Delay-Tolerant Netw., 2005,pp. 252-259.

[3] V. Cerf, S. Burleigh, A. Hooke, L. Torgerson, R. Durst, K. Scott, K. Fall, and H.Weiss, Delay-tolerant networking architecture. RFC 4838. [Online]. Available: ftp://ftp.rfc-editor.org/in-notes/rfc4838.txt

[4] T. Spyropoulos, K. Psounis, and C. S. Raghavendra, "Efficient routing in intermittently connected mobile networks: The single-copy case," IEEE/ACM Trans. Netw., vol. 16, no. 1, pp. 63-76, Feb. 2008.

[5] A. Vahdat and D. Becker, "Epidemic routing for partially connected ad hoc networks," Duke Univ., Durham, NC, Tech. Rep. CS-2000-06, 2000.

[6] R. Bruno and M. Nurchis, "Survey on diversity-based routing in wireless mesh networks: Challenges and solutions," Comput. Commun., vol. 33, no. 3, pp. 269282, Feb. 2010.

[7] H. Füler, J. Widmer, M. Käsemann, M. Mauve, and H. Hartenstein, "Contention-based forwarding for mobile ad hoc networks," Ad Hoc Netw., vol. 1, no. 4, pp. 351369, Nov. 2003.
[8] M. Heissenbüttel, T. Braun, T. Bernoulli, and M. Wälchi, "BLR: Beaconless routing algorithm for mobile ad hoc networks," Comput. Commun., vol. 27, no. 11, pp. 10761086, Jul. 2004.

[9] R. R. Choudhury, "Brownian gossip: Exploiting node mobility to diffuse information in ad hoc networks," in Proc. Int. Conf. Collaborative Comput.: Netw., Appl. Worksharing, 2005, pp. 1-5.

[10] T. Hara and S. K. Madria, "Consistent Management Strategies for Data Replication in Mobile Ad hoc Networks," IEEE Transactions on Mobile Computing, vol. 8, no. 7, July 2009, pp. 950-967.

[11] N. Aschenbruck, E. Gerhards-Padilla, and P. Martini, "Modeling mobility in disaster area scenarios," Perform. Eval., vol. 66, no. 12, pp. 773-790, Dec. 2009.

[12] M. Asplund and S. Nadjm-Tehrani, "A partition-tolerant manycast algorithm for disaster area networks," in Proc. 28th Int. Symp. Reliable Distrib. Syst., 2009, pp. 156165 .

[13] M. B. Donley and N. A. Schwartz, United States Air Force Unmanned Aircraft Systems Flight Plan 20092047, 2009. [Online]. Available: http://handle.dtic.mil/100.2/ADA505168

[14] P.-C. Cheng, K. C. Lee, M. Gerla, and J. Härri, "GeoDTN +Nav: Geographic DTN routing with navigator prediction for urban vehicular environments," Mobile Netw. Appl., vol. 15, no. 1, pp. 61-82, Feb. 2010.

[15] J. LeBrun, C. Chuah, D. Ghosal, and M. Zhang, "Knowledge-based opportunistic forwarding in vehicular wireless ad hoc networks," in Proc. IEEE 61st Veh. Technol. Conf., 2005, pp. 2289-2293.

[16] S. M. Das, H. Pucha, and Y. C. Hu, "Performance comparison of scalable location services for geographic ad hoc routing," in Proc. IEEE 24th Annu. Joint Conf. IEEE Comput. Commun. Soc., 2005, pp. 1228-1239. 